\title{
From the optic tectum to the primary visual cortex: migration through evolution of the saliency map for exogenous attentional guidance
}

\author{
Li Zhaoping \\ Department of Computer Science, University College London, UK \\ Current Opinion in Neurobiology, 2016, 40:94-102
}

\begin{abstract}
:
Recent data have supported the hypothesis that, in primates, the primary visual cortex (V1) creates a saliency map from visual input. The exogenous guidance of attention is then realized by means of monosynaptic projections to the superior colliculus, which can select the most salient location as the target of a gaze shift. V1 is less prominent, or is even absent in lower vertebrates such as fish; whereas the superior colliculus, called optic tectum in lower vertebrates, also receives retinal input. I review the literature and propose that the saliency map has migrated from the tectum to V1 over evolution. In addition, attentional benefits manifested as cueing effects in humans should also be present in lower vertebrates.
\end{abstract}

\section{Highlights}

- A saliency map in the primary visual cortex for primates.

- A saliency map in the optic tectum for archer fish.

- Through evolution, the saliency map migrated from the optic tectum to the primary visual cortex.

\section{Introduction}

The saliency of a visual location, at least as we define it here, is the degree to which this location attracts attention or gaze exogenously. Exogenous guidance is reflexive or bottom-up, driven by external rather than internal factors (such as the goal of an on-going task), which are endogenous or top-down. For example, the location of an orientation singleton, such as a vertical bar in a background of horizontal bars, is salient or has a high saliency value; so is the location of a color singleton, such as a red dot among many green ones. Such feature singletons, examples of which are shown in Fig. 1a, are so salient that they are said to pop-out perceptually. Saliency at a location in an image can be measured by the brevity of the reaction time (RT) taken to saccade towards, or find a target at, this location. In humans and monkeys, guiding attention exogenously to a spatial location, such as by flashing a brief cue at this location, increases the speed and accuracy of detecting, recognizing, or discriminating visual inputs appearing subsequently at the cued location[1 $\left.{ }^{\bullet}, 2\right]$. This attentional benefit is called the cueing effect and can also be used to measure the saliency of the cue.

Traditional views[3] presume that it is higher brain areas, such as the frontal eye field (FEF) in humans, that contain a saliency map of the visual world to guide attention exogenously. This was partly inspired by the observations that saliency is a general property that could arise from visual inputs with any feature values (e.g., vertical or red) in any feature dimension (e.g., color, orientation, and motion) whereas neurons in lower visual areas such as the primary visual cortex 
(a) salient singleton in orientation, color, or in both color and orientation
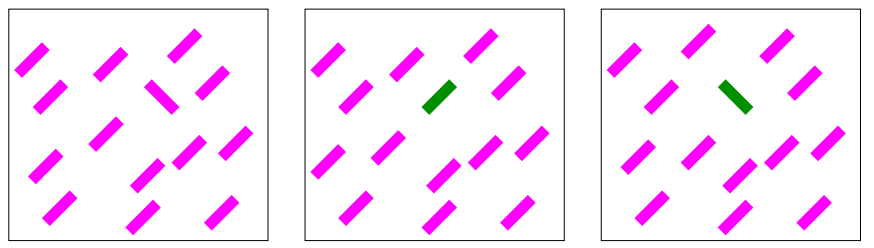

(b) the unique cross is more salient than the unique bar

(c) A schematic of how a saliency map in V1 guides gaze shift
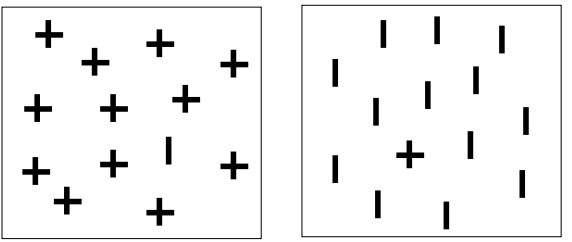

$\uparrow$ To visual cortical areas downstream

Retinotopic V1 neural responses visualized by the sizes of the dots

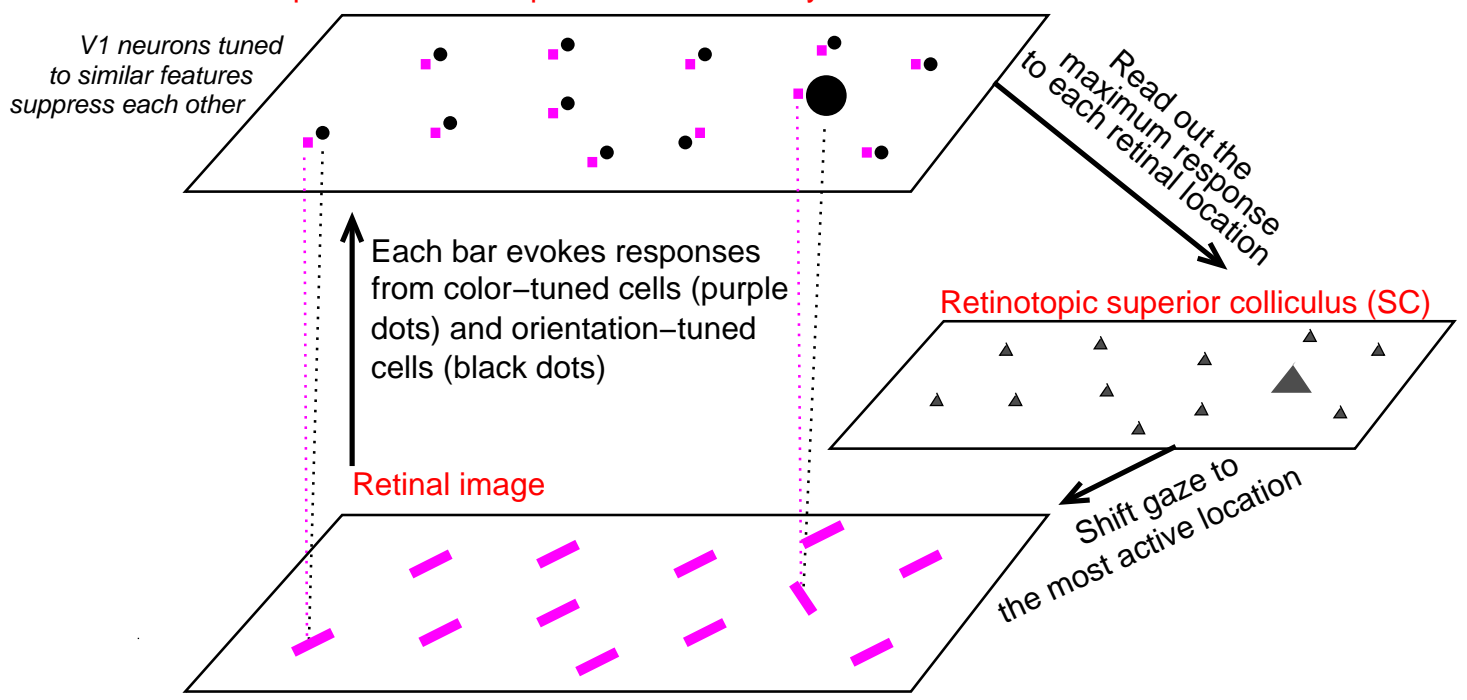

Figure 1: Visual saliency and its neural mechanisms in primates. (c) is from Figure 1 of [9].

(V1) are tuned to specific feature values (e.g., the vertical orientation) rather than being feature untuned. However, recent behavioral data in humans, combined with physiological knowledge, support the hypothesis that V1 creates the saliency map $\left[4,5^{\bullet \bullet}\right]$. Meanwhile, FEF is often considered to be responsible for the different function of endogenous attentional guidance.

Although the idea of computing saliency without frontal/parietal brain areas contradicts traditional wisdom, it is perhaps more appealing when considering lower mammals such as rats or non-mammalian vertebrates such as fish. All vertebrates should have visual attentional mechanisms, as attention helps to focus brain's limited processing power to a fraction of visual inputs; however, prefrontal cortical areas (which include FEF) comprise a smaller fraction of the whole cortex $[6,7]$ in lower mammals, and fish lack neocortex. For such lower vertebrates, it appears that the superior colliculus (SC) (called optic tectum (OT) in non-mammals) is involved in visual (attentional) orienting[8].

Note that saliency at a location of the same visual item is context dependent, for example, a vertical bar is salient among many horizontal bars but not among other identical vertical bars. Hence building a saliency map requires analyzing visual features (such as color, orientation, and motion direction) and comparing features at different visual locations. Fig. 2a outlines the essential building blocks for generating and utilizing the saliency map in vision. To generate the map, 
some degree of visual analysis is necessary to, first, build feature tuning in neurons and to, second, compute saliency by additional processing of the responses from feature tuned neurons, effectively comparing features at different visual locations. For the saliency map to impact behavior, there should be (1) a winner-take-all (WTA) computation over the saliency map to identify the most salient location (when the saliency map is combined with endogenous factors and other sensory input, the net outcome is what is called a priority map[10, 11], and WTA will then identify the location of the highest priority); and (2) either overt or covert orienting towards the winning location (for simplicity, we omit avoidance behavior). In overt orienting, the attended location is shifted to the center of the visual field or is acted upon/towards (e.g., in predatory behavior). Both covert and overt orienting impact visual analysis by, for example, gain controls to enhance neural responses or sensitivities $[2,11,12]$, to lead to the cueing effects.

The location of the WTA computation appears to be conserved over evolution. Across vertebrates[13 ••, 7], SC/OT is retinotopic. Its upper layers receive visual inputs (from the retina and forebrain/V1); its intermediate and deep layers receive inputs from the upper layers and receive context inputs, which include (spatially aligned) sensory inputs of non-visual modalities and other inputs from the forebrain (such as endogenous inputs from FEF in primates) $\left[13^{\bullet \bullet}, 7\right]$. WTA appears to occur in the intermediate and deep layers of SC/OT, activities at different visual locations suppress each other so that the response to the winner location is higher than the responses to the other locations $\left[13^{\bullet \bullet}, 14^{\bullet \bullet}, 15^{\bullet}, 7,16^{\bullet}, 17^{\bullet \bullet}, 18\right]$. Along with orienting to the attended location (e.g., by directing a saccade), the outcome of WTA can impact visual analysis in at least two further ways via efferent projections from SC/OT. One of these innervates dorsal thalamic regions (such as pulvinar and lateral geniculate nucleus (LGN), which in turn project to cortex or to forebrain areas in non-mammals. The second efferent projection is direct to the retina in lower vertebrates[7].

This paper argues that the brain region that realizes the saliency map is not conserved across species. I will review recent findings which suggest that V1 could realize a saliency map which guides attention exogenously in primates, and propose that, over the course of evolution, the saliency map migrated from the OT/SC to V1, just as much visual analysis migrated from subcortical areas to the cortex.

To avoid confusion, we must first clarify what is meant by the claim that a saliency map arises in a particular brain region. This is that the saliency values are first computed and explicitly represented in neural responses in this brain region. Downstream brain regions can inherit the saliency values for further processing or action. For example, when this map is first created in V1, it can be projected directly or indirectly to SC or FEF. In turn, they can read out the saliency values to execute a shift in attention or arrange for exogenous and endogenous influences over attention to compete or be combined. Although the read-out makes a copy of the saliency map in downstream areas, these downstream areas are not deemed responsible for the saliency map, just as a printer attached to a computer is not responsible for any graphics sent from the computer for printing. SC neurons in monkeys are normally untuned to any visual feature[13 $\left.{ }^{\bullet \bullet}\right]$, making the monkey SC a relatively pure map of space that is devoid of visual feature analysis. Of course, such a pure space map is appropriate for performing a WTA operation on a saliency map read out from an upstream source. 


\section{Saliency maps in vertebrate species}

\section{A saliency map in V1 for primates}

Noting that the activities of V1 neurons could serve as a universal currency to bid for attention regardless of those neurons' preferred features, $\mathrm{Li}\left[4,5^{\bullet \bullet}\right]$ proposed that the saliency map is created in V1. According to this, the saliency at any location in a scene is the highest V1 response to input at that location relative to the highest responses to other locations, see Fig 1c. The neural mechanism by which V1 responds more vigorously to salient feature singletons than to the non-salient background items is iso-feature suppression[19 $\left.{ }^{\bullet}, 4\right]$, which makes V1 neurons tuned to the same or similar features suppress each other. For example, nearby V1 neurons tuned to the same orientation, for example, right-tilt, suppress each other. Thus, in an image containing many background right-tilted bars (Fig 1c), the responses to these background bars are lower than the response to the salient singleton, the unique left-tilted bar, because the V1 neuron responding to this orientation singleton escapes iso-orientation suppression. Similarly, iso-color suppression makes salient a color singleton, like that in Fig 1a.

Data supporting this V1 saliency hypothesis have since emerged. In particular, behavioral data confirmed its surprising prediction that an eye-of-origin singleton should be very salient. This singleton is for example an item uniquely shown to the left eye among many items shown to the right eye. Such a singleton, if it is unique only in terms of its eye-of-origin feature, can scarcely be distinguished in its appearance from the surrounding visual items. Nevertheless, it is no less salient in terms of its ability to attract gaze than a salient and highly distinctive orientation singleton[20 $0^{\circ}$, 21]. This finding provides a hallmark that the saliency map is in V1 rather than extrastriate cortical areas, since cortical neurons outside V1 (unlike those in V1) are not tuned to eye-of-origin[22, 23] and hence cannot contribute to a saliency computation based on this feature. Indeed, it is the insensitivity of the extrastriate cortical neurons to the eye-of-origin that makes this feature (unlike orientation, color, or motion direction) invisible to perception.

V1 projects monosynaptically and retinotopically to the upper layers of the superior colliculus (SC) $\left[13^{\bullet}, 24,2^{\bullet}\right]$. This is likely how the saliency map is copied from V1 to SC, see Fig 1c. Since the saliency value of a location is represented by the highest $\mathrm{V} 1$ response to this location regardless of the preferred features of the V1 neurons, some sort of neural circuitry is needed to convey this saliency value from V1 to SC without projecting the feature tuning property. For example, V1 circuit could be such that each SC projecting layer 5 neuron combines local neural responses such that its response is equal to the largest response from the other V1 neurons sharing its receptive field location. Another possibility is that both the circuitry around layer 5 in V1 and the circuit inside the SC onto which the V1 fibers project and converge play a role in copying the saliency map[11]. In either case, in V1, the SC projecting layer 5 neurons, compared to the other layer 5 neurons, should be less tuned to visual features. Meanwhile, SC neurons at different locations suppress each other; this suppression enables a WTA competition in the saliency map copied from V1 to select the most salient location[14*0]. Visual orienting towards the most salient location can be made by the activation of SC neurons in its deeper, motor-related, layers. These SC neurons project to the brainstem, and directly or indirectly to the spinal cord, to shift gaze and/or to turn head and body $\left[13^{\bullet}, 26^{\bullet}, 7\right]$ to the selected location.

If SC is lesioned in monkeys, saccades can be directed via a longer route through FEF, which can bypass SC and guide saccades itself directly via the brainstem[26 $6^{\circ}$. Consequently, a SC lesion prolongs RTs to saccade to a highly visible object that suddenly appears on a blank screen, and eliminates the so-called express saccades that have the shortest $\mathrm{RTs}\left[27^{\bullet}\right]$. This is because the lesion 
impairs the exogenous, reflexive, control route realized by the SC. On the other hand, lesions in FEF, which is associated with endogenous attentional guidance, only weakly affect such saccadic $\mathrm{RTs}\left[27^{\circ}\right]$ since reflexive orienting via SC is sufficient for such easy tasks. Conversely, FEF lesions make memory-guided saccades (which are endogenous and non-reflexive) difficult[28]. Primate retina also projects to SC; however this appears not to constitute a saliency map (at least in normal circumstances). This is because lesioning $\mathrm{V} 1$ while leaving the retina intact makes monkeys

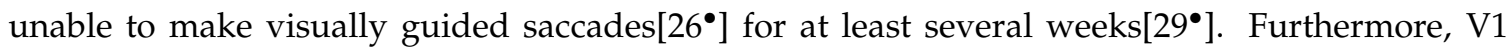
lesions make monkey SC neurons in the (motor-related) deeper layers non-responsive to visual inputs[26 $\left[26^{\bullet}\right.$.

Since neurons in V1 are only tuned to simple features like bars and edges, one may wonder whether saliencies at locations of complex visual objects such as faces may require at least partial contributions from extrastriate visual areas such as V4 and IT. Faces are indeed likely to attract attention by a combination of both fast and transient exogenous attraction and slower and longer lasting endogenous factors[30, 31]. However, exogenous attentional attraction does not require object recognition, thus not having face detectors in V1 cannot be an argument against V1's role in the saliency of a visual location on a face. A case of looking or attending before seeing or recognizing the visual object at the attended location is dramatically demonstrated in the strong saliency of the eye-of-origin singleton whose unique eye-of-origin feature is not recognized[20••].

Not only is recognition unnecessary for saliency, recognition of visual object by itself is insufficient to account for saliency. Fig $1 \mathrm{~b}$ demonstrates that a cross among bars is more salient than a bar among crosses; this would not be the case if saliency depended on recognizing the bar, the cross, and thus the difference between the bar and cross, since this difference is the same in both images of Fig 1b. Instead, V1 saliency hypothesis suggests that the location of the unique cross is salient not because any V1 neuron detects or recognizes the cross, but rather because the neurons responding to the unique horizontal bar in the cross escape iso-orientation suppression and respond more vigorously than neurons responding to the other (vertical) bars in the image.

It has also been shown that behavioral RTs to the feature singletons like those in Fig 1a can be fully and quantitatively accounted for by the predictions from the V1 saliency hypothesis without evoking any extrastriate contribution[9]. For example, the color-orientation singleton (unique in both color and orientation) in Fig 1a is so salient that the RT to detect it is shorter than the RT to the color singleton and the RT to the orientation singleton (each unique in only one feature dimension) in Fig 1a. Furthermore, this RT to the color-orientation singleton is also shorter than predicted by a statistical facilitation between two independent processes, one for the RT to the color singleton and the other for the RT to the orientation singleton[32, 33॰]. This finding manifests the existence of V1 neurons tuned simultaneously to both color and orientation[34]. Furthermore, it also manifests that such V1 neurons must have sufficient activations (compared to the single-feature tuned V1 cells) and exhibit iso-feature suppression in both feature dimensions such that their responses to their preferred input features are highest when this input feature is a feature singleton in both feature dimensions $\left[33^{\bullet}, 35\right]$.

\section{A saliency map in the optic tectum for archer fish}

Archer fish hunt by shooting water from their mouths at insects flying above water. Recently, Ben-Tov et al. [36 ${ }^{\bullet}$ ] found evidence for a saliency map in OT of this species. Singletons in motion direction or motion speed feature (analogous to the color or orientation feature in Fig 1a) apparently pop-out for archer fish such that these fish preferentially orient to singletons rather than background items and their orienting RTs are independent of the number of background items. 
(a) Building blocks for creating and utilizing the saliency map for exogenous guidance of attention

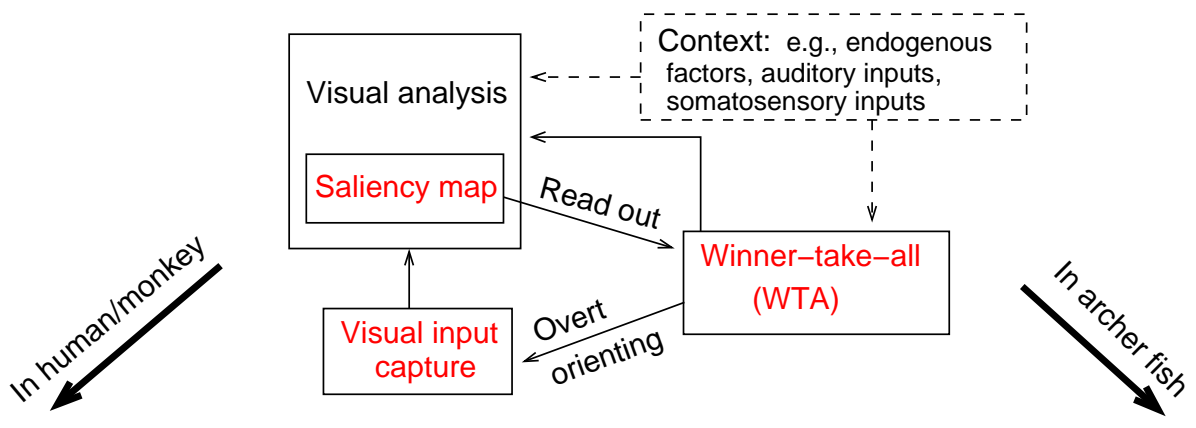

(b) the saliency map in $\mathrm{V} 1$

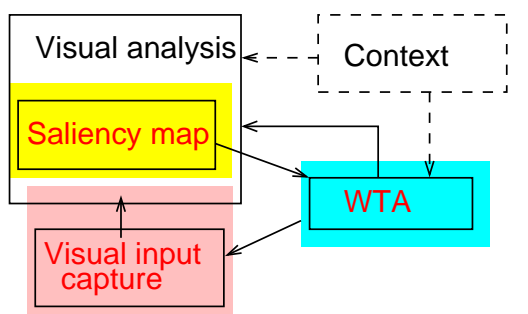

V1 Retina (c) the saliency map in optic tectum

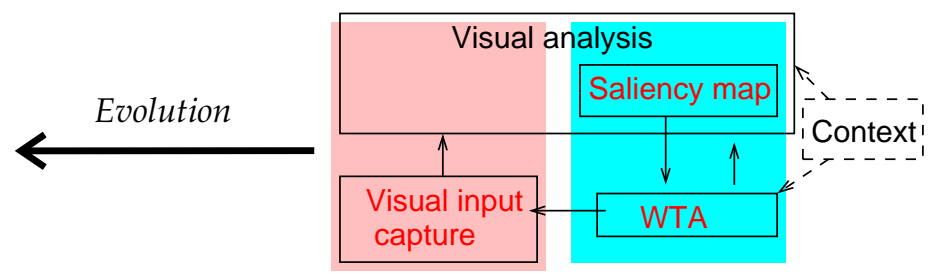

Figure 2: Building blocks for the creation and utilization of the saliency map in the brain (a) and its implementations (b) and (c) in animal species. The pink, yellow, and cyan colored regions denote retina, $\mathrm{V} 1$, and SC/OT, respectively.

Furthermore, a direction-speed singleton, unique in both motion direction and motion speed, is even more salient than a singleton unique in only direction or speed[36 $\left.{ }^{\bullet \bullet}\right]$.

The OT neurons in archer fish, like V1 neurons in monkeys and cats, are tuned to orientation and/or motion direction[37], and, based on their tunings to spatiotemporal frequencies[37], some neurons should also be tuned to motion speed. Focusing on the feature dimensions of motion direction and motion speed, Ben-Tov et al. [36 $\left.6^{\bullet}\right]$ found that many OT neurons in archer fish showed iso-feature suppression. OT neural responses to their preferred feature are higher when this feature is a singleton rather than a background item, and, for some neurons, this response is higher still when the singleton is unique in both feature dimensions $\left[36^{\bullet \bullet}\right]$. These OT neural properties are analogous to those in primate $\mathrm{V} 1$ which has cells tuned (for example) to color, orientation, or both and exhibits the corresponding iso-feature suppression. The link between visual search behavior and V1 physiology in primates mirrors that between behavior and OT physiology in archer fish, although, for archer fish, whether the extra degree of saliency in the direction-speed (double-feature) singleton is beyond that due to a statistical facilitation between the two feature dimensions has yet to be determined. Taken together, these findings suggest that the OT of archer fish performs the sort of saliency computation that V1 does in primates.

Could the saliency map in OT be inherited from a V1 homolog in fish? Fish lack a neocortex; and it is not known if there is any homolog of V1 in fish forebrain, which is typically concerned much more with olfaction than vision[7]. We suggest that it is also unlikely that the saliency map in OT is inherited from the fish retina, because of the feature selectivities of the OT neurons. To see this, note first that, in primates, the saliency map in V1 is copied to the SC, whose neurons are 
mostly untuned to visual features[13*0]. In other words, while feature-tuned neurons help build a saliency map, copying this map from one brain region to another does not require copying the feature-tuning properties. Not only are the OT neurons in fish feature-tuned, but it has also been found that, in zebrafish at least, feature tuning in OT can be very different from that in the retina (although it can also be inherited from that in the retina) $\left[38,39^{\circ}, 40\right]$. This suggests that neural feature tuning in fish OT serves its own purpose, perhaps particularly to create a saliency map.

\section{Neural processes for saliency computation across different vertebrates}

Based on the observations in the previous sections, we consider the hypothesis that monkey and fish lie at the two ends of the vertebrate phylogenetic scale, with saliency maps in V1 and the upper layers of OT, respectively, see Fig. 2bc. To evaluate this hypothesis, we examine the trend across vertebrates of visual processing leading to the saliency map.

\section{Relative emphasis between retinothalamic and retinotectal pathways}

From lower to higher vertebrates, the percentage of resources devoted to SC/OT compared with that devoted to V1 is reduced. One way to quantify this is via the ratio between the volume of SC and that of LGN, which relays retinal input to V1. This decreases from lower to higher mammals: it

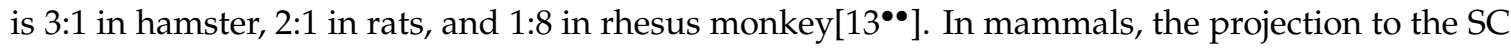
from the forebrain is often more extensive than that from the retina, but such projections are sparse in non-mammalian species[13 $\left.{ }^{\bullet}\right]$. Meanwhile, from lower to higher mammals, the percentage of retinal ganglion cells that project to the SC/OT decreases[13 ${ }^{\bullet \bullet}$ ], from almost $100 \%$ in rabbit[41] and

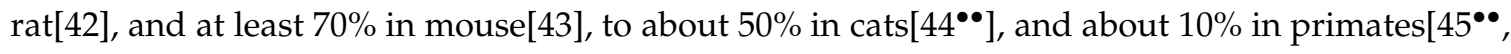
$\left.44^{\bullet \bullet}\right]$.

\section{Degree of visual feature tuning in tectum neurons}

Meanwhile, in higher vertebrates, feature tuning in SC/OT neurons appears weaker. The OT of fish, amphibians, and reptiles are replete with pyramidal cells with extensive basal and apical dendritic arborizations that allow these cells to sample retinal inputs for visual analysis[13 $\left.{ }^{\bullet \bullet}\right]$. However, in mammals, the SC of cat does not have either pyramidal cells or cells with extensive dendritic arbors[13*0]. Consistent with this, single cells in the colliculus show more specificity for patterns in fish, amphibians, and birds than in higher mammals[13 $\left.\bullet^{*}, 46\right]$. OT/SC cells tuned to orientation have been seen in ground squirrel, tree shrew [13 ${ }^{\bullet \bullet}$, and mouse[47, 48], but not in cat

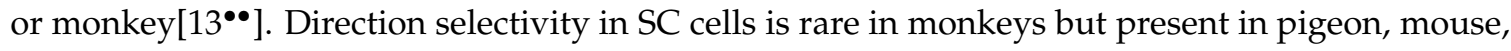
hamster, and cat[13 $\left.{ }^{\bullet}, 47\right]$. After removing inputs from V1 to SC, this direction selectivity in SC cells persists in mouse[47] but is reduced in cats $\left[13^{\bullet \bullet}, 45^{\bullet \bullet}\right]$, suggesting that this feature is partly inherited from V1 in cats. Upper layer SC neurons in mouse also display tuning to the feature of direction-independent motion, looming motion, speed, and size[49].

That OT/SC neurons are feature tuned perhaps helps them to compute saliency through the mechanism of iso-feature suppression, and iso-feature suppression is consistent with neural response properties observed in archer fish[36 $\left.{ }^{\bullet \bullet}\right]$. However, although feature singletons such as those in orientation, color, motion direction, and eye-of-origin features are highly salient for humans, in

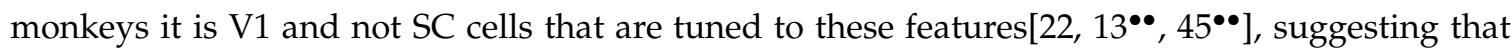
saliencies for these singletons are calculated in V1 before being projected to SC. 


\section{Retinal ganglion cells and their central projections}

In cats and monkeys, the majority of retinal ganglion cells (RGCs) are $\mathrm{X}$ and midget cells respectively. They have spatially center-surround receptive fields, respond linearly to inputs, and are not tuned to complex spatiotemporal features like orientation and motion direction. They project to $\mathrm{LGN}$ en route to $\mathrm{V} 1$ and do not project to $\mathrm{SC}\left[44^{\bullet \bullet}\right]$. Accumulating evidence supports the idea that these RGCs code visual input information efficiently for transmission to the brain[50,11]. The second most numerous type of RGC cells is the Y or parasol cells for cat or monkey, respectively. These are similar to the $X$ or midget cells, but are more sensitive, have nonlinear and more transient responses to inputs, have larger receptive fields, and they project to both LGN and SC[44**]. One suggestion is that they send the minimum necessary amount of information to the brain as fast as possible[51].

However, lower vertebrates have some dozens of other types of RGCs[44* $]$, and RGCs of these other types collectively comprise an increasing percentage of the total number of RGCs. Many of these RGC types have complex spatiotemporal receptive fields and respond nonlinearly to inputs, such that they can be viewed as detectors for such spatiotemporal features as orientation, motion direction, foreground motion, and local edge contrast $\left[44^{\bullet \bullet}, 52,53,40,54\right]$. Let us call them the feature-detector RGCs. They tend to have larger receptive fields and project to

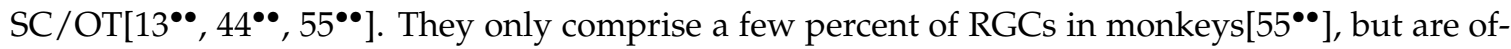
ten in the majority in lower vertebrates[44 $\left.{ }^{\bullet}, 40,52,54\right]$. For example, in the mouse retina, about $50 \%$ of the RGCs are feature detectors for motion direction, orientation, or local edge contrast $\left[56^{\circ}\right]$, and there are 20 or more RGC types, depending on the methods of classification[54, 52, 56 $6^{\circ}$. Birds, whose visual system is dominated by the retina-to-OT rather than the retina-to-thalamus pathway [57, 58], also have a heterogenous collections of RGC types, as characterized by the morphology of dendritic fields of the RGCs[58,59]. Furthermore, the bird RGCs that project to the thalamus tend to have the less complex, center-surround, structure for their receptive fields[58, 59].

Hence, in vertebrates, feature-detector RGCs tend to project to SC/OT while RGCs of simpler (e.g., center-surround) receptive fields tend to project to thalamus, with a trend for an increasing emphasis on the retina-to-tectum pathway in lower vertebrates. This is consistent with the idea that the saliency map is built in SC/OT in lower vertebrates, based partly on the ready-made feature maps from retina and partly from additional analysis by SC/OT's own feature detectors (e.g., in pigeon $\left[60^{\circ}\right]$ and zebrafish $\left.\left[39^{\circ}\right]\right)$. Meanwhile, although RGCs that project to thalamus have simpler receptive fields, monkey V1 receiving these efficiently coded visual inputs can use them to construct neurons tuned to many different features, and thereby build a richer saliency map[11].

One excellent example of the latter is that cells in the monkey retina that are tuned to color (the midget cells) do not project to $\mathrm{SC}\left[13^{\bullet \bullet}, 44^{\bullet \bullet}\right]$. However, color singletons enjoy a substantial saliency. According to our scheme, this saliency necessarily depends on V1 inputs to SC.

\section{V1 processing}

While there is no known V1 homolog in fish, V1's role in visual processing increases in higher vertebrates. Selectivities of V1 neurons to orientation, motion direction, and scale are present across mammals such as monkeys[22], cats[61], and mouse[62, 63], while visual acuity in monkey is 100 times higher than that in mouse. In mice, although selectivities in orientation and direction features are already prevalent in retina, recent data suggest that these selectivities are further refined in V1 so that the cortical neurons exhibit sharper tunings and have a greater diversity of preferred features[64]. 
In visual Wulst, which is the homolog of V1 in birds, orientation selectivity has been observed in chickens [65], and selectivity to orientation, motion direction, and even binocular disparity have been observed in owls[66]. The size of the receptive fields in chicken Wulst is huge, in the range of $20-45^{\circ}[65]$, slightly larger than those in mouse[63], but receptive field sizes in owls are heterogenous with some as small as about one degree[66].

\section{Effects of lesion in V1 or SC/OT}

In birds, OT lesions produce deficits in visual discrimination, localization, and reflex orientation[13 $\left.{ }^{\bullet \bullet}\right]$ without recovery (in pigeons) even after extensive postoperative training[67]. In frogs, an OT lesion makes the animal blind except for ballistic escape behavior[13*0]. In fish and amphibians in general, ablating OT causes substantial deficits in visual pattern perception[13 $\left.{ }^{\bullet \bullet}, 26^{\bullet}\right]$. By contrast, in mammals such as cats, a SC lesion mainly affects orienting $\left[13^{\bullet \bullet}, 2^{\bullet}\right]$. Cats become less easily distracted, although there are some reports of deficits in pattern perception[13 $\left.{ }^{\bullet \bullet}\right]$. In monkeys, as already mentioned, a SC lesion does not noticeably affect behavior except for eliminating the short

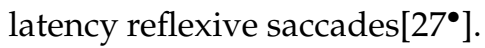

The opposite trend arises for lesions or disruptions in V1. In monkeys, as mentioned, they render deep layer SC cells unresponsive to visual inputs $\left[13^{\bullet \bullet}, 26^{\bullet}\right]$ and make the animal unable to make visually guided saccade for at least weeks $\left[26^{\bullet}, 2^{\bullet}\right]$, although the animal can still fixate when

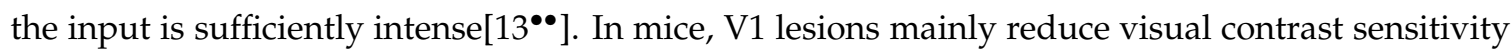
and acuity in visual discrimination tasks $[68,69]$. While SC neurons in monkeys are mostly untuned to visual features[13 ${ }^{\bullet \bullet}$, disruption of the V1 to SC pathway reduces or eliminates direction selectivity in SC (upper layer) neurons in cats and hamsters[13 $\left.{ }^{\bullet \bullet}, 49\right]$ but not in ground squirrels $\left[13^{\bullet \bullet}\right]$, rabbits[13 $\left.{ }^{\bullet}\right]$, and mice[47, 49]. These findings collectively suggest that visual orientation involves SC/OT in all animals, while visual analysis relies more on SC/OT and V1, respectively, for lower and higher vertebrates.

\section{Concluding remarks}

In summary, it appears that through the evolutionary expansion of the forebrain, visual analysis

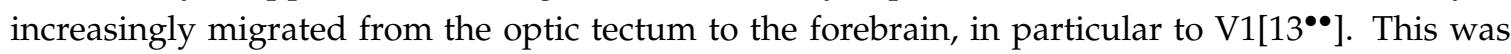
accompanied by decreases in the percentage of retinal ganglion cells that project to the tectum. It was also accompanied by increases in the percentage of retinal ganglion cells which, like the midget cells in monkey, are not special feature detectors, but rather encode visual information efficiently for transmission via the thamalus to the forebrain to enable visual analysis. With the migration of visual analysis to V1, the computation of the saliency map also migrated from the tectum to V1 in monkeys. This saliency map is then read out by the tectum to execute WTA for attentional selection.

Could some vertebrates have a saliency map in retina? This is perhaps unlikely. In higher vertebrates, the saliency map could not be built in the retina from the limited feature analysis that is present there. The retina of lower vertebrates does include much richer feature analysis. However, building a saliency map in the retina before copying it to the tectum would only make sense if no substantial visual feature analysis was additionally needed in the tectum. However the tectum in lower vertebrate occupies a larger fraction of the brain and performs substantial visual feature analysis.

In traditional wisdom for primate vision, $\mathrm{V} 1$ has been seen as too lowly to play a significant role in guiding attention, a task presumed for the frontal and parietal brain areas[3]. It is helpful 
to note that, from the perspective of a frog, who could be easily fooled to attack an imitation fly in its visual field, V1 could be seen as an overkill for guiding its visual orienting. This perspective reminds us that, despite our common sense, exogenous attentional guidance does not require visual object recognition.

This paper assumes that the drive behind essential behavior of visual orienting in lower vertebrates has basically the same nature as exogenous attentional guidance in humans and monkeys. This assumption predicts that the benefits of attentional guidance manifested in the cueing effects observed in humans and monkeys $\left[1^{\bullet}, 2,12\right]$ should be similarly present in lower vertebrates such as frogs. This prediction can be readily tested, and is consistent with recent observations in birds[70].

Acknowledgement This work is supported by the Gatsby Charitable Foundation. I would like to thank Tom Baden, David Berson, Onkar Dhande, Marcus Meister, Ronen Segev, and Ben Sivyer for helping with the literature, and Peter Dayan for comments on the paper.

\section{References}

- [1] Posner MI: Orienting of attention. Quarterly Journal of Experimental Psychology 1980, 32:325.

Behavioral observations in humans of the cueing effects by attentional guidance

[2] Carrasco M: Visual attention: The past 25 years. Vision research 2011, 51:1484-1525.

[3] Itti L, Koch C: Computational modelling of visual attention. Nature Reviews Neuroscience 2001, 2:194-203.

[4] Li Z: Contextual influences in V1 as a basis for pop out and asymmetry in visual search. Proceedings of the National Academy of Sciences of the USA 1999, 96:10530-10535.

$\bullet \bullet$ [5] Li Z: A saliency map in primary visual cortex. Trends in Cognitive Sciences 2002, 6:9-16.

An early paper on the hypothesis that $\mathrm{V} 1$ in primates creates a saliency map

[6] Fuster JM: Frontal lobe and cognitive development. Journal of neurocytology 2002, 31:373385.

[7] Butler AB, Hodos W: Comparative Vertebrate Neuroanatomy: Evolution and Anatomy. John Wiley \& Sons, Inc.; 2005.

[8] White BJ, Munoz DP: The superior colliculus. In The Oxford Handbook of Eye Movements, Edited by Liversedge SP, Gilchrist I, Everling S, Oxford University Press; 2011, 195-214.

[9] Zhaoping L, Zhe L: Primary visual cortex as a saliency map: A parameter-free prediction and its test by behavioral data. PLoS Comput Biol 2015, 11:e1004375.

[10] Bisley J, Goldberg M: Attention, intention, and priority in the parietal lobe. Annual Review of Neuroscience 2011, 33:1-21.

[11] Zhaoping L: Understanding Vision: theory, models, and data. Oxford University Press; 2014.

[12] Wang F, Chen M, Yan Y, Zhaoping L, Li W: Modulation of neuronal responses by exogenous attention in macaque primary visual cortex. The Journal of Neuroscience 2015, 35:1341913429. 
-• [13] Schiller PH: The superior colliculus and visual function. In Handbook of Physiology, Section 1: The Nervious System; Vol. III, Sensory Processes, Part 1, DOI:10.1002/cphy.cp010311, Edited by Brookhart J, Mountcastle V, Darian-Smith I, Geiger S, Wiley Online Library; 1984, 457-505. 1984.

A comprehensive review of superior colliculus across animal species

•• [14] Munoz DP, Istvan PJ: Lateral inhibitory interactions in the intermediate layers of the monkey superior colliculus. Journal of Neurophysiology 1998, 79:1193-1209.

Recording from and stimulation of the SC neurons while the animal performed fixation or saccading tasks, suggested local inhibition circuit in the intermediate layer of SC for WTA computation

- [15] McPeek RM, Keller EL: Saccade target selection in the superior colliculus during a visual search task. Journal of Neurophysiology 2002, 88:2019-2034.

Recording of SC neurons in monkeys doing a visual search task, showing that the later phase responses of some neurons are higher when the search target, rather than non-target, is in the receptive field of the neurons, manifesting competition in SC circuit

- [16] Mysore SP, Asadollahi A, Knudsen EI: Signaling of the strongest stimulus in the owl optic tectum. The Journal of Neuroscience 2011, 31:5186-5196.

Attentional guidance by the optic tectum in owl

$\bullet \bullet$ [17] Knudsen EI: Control from below: the role of a midbrain network in spatial attention. European Journal of Neuroscience 2011, 33:1961-1972.

A review comparing the SC of mammals and OT of birds

[18] Vokoun CR, Jackson MB, Basso MA: Intralaminar and interlaminar activity within the rodent superior colliculus visualized with voltage imaging. The Journal of Neuroscience 2010, 30:10667-10682.

- [19] Allman J, Miezin F, McGuinness E: Stimulus specific responses from beyond the classical receptive field: neurophysiological mechanisms for local-global comparisons in visual neurons. Annual Review of Neuroscience 1985, 8:407-30.

A review that visual cortical neural responses exhibit contextual influences, in particular, iso-feature suppression

-• [20] Zhaoping L: Attention capture by eye of origin singletons even without awareness-a hallmark of a bottom-up saliency map in the primary visual cortex. Journal of Vision 2008, 8:article 1.

Paper on the dramatic saliency of the eye-of-origin singleton in human

[21] Zhaoping L: Gaze capture by eye-of-origin singletons: Interdependence with awareness. Journal of Vision 2012, 12:article 17.

[22] Hubel DH, Wiesel TN: Ferrier lecture: Functional architecture of macaque monkey visual cortex. Proceedings of the Royal Society of London Series B, Biological Sciences 1977, 198:1-59. 
[23] Burkhalter A, Van Essen DC: Processing of color, form and disparity information in visual areas vp and $\mathrm{V} 2$ of ventral extrastriate cortex in the macaque monkey. The Journal of Neuroscience 1986, 6:2327-2351.

[24] Lock T, Baizer J, Bender D: Distribution of corticotectal cells in macaque. Experimental brain research 2003, 151:455-470.

- [25] Cerkevich CM, Lyon DC, Balaram P, Kaas JH: Distribution of cortical neurons projecting to the superior colliculus in macaque monkeys. Eye Brain 2014, 2014:121-137.

Showing that V1 provides the most corticotectal projections to SC in monkeys

- [26] Schiller P: The neural control of visually guided eye movements. In Cognitive Neuroscience of Attention, a Developmental Perspective, Edited by Richards JE, Lawrence Erlbaum Associates, Inc., Mahwah, New Jersey, USA; 1998, 3-50. 1998.

A comprehensive review of the control of eye movements by SC, FEF, and visual cortices in monkeys and cats

- [27] Schiller PH, Sandell JH, Maunsell JH: The effect of frontal eye field and superior colliculus lesions on saccadic latencies in the rhesus monkey. Journal of Neurophysiology 1987, 57:1033-1049.

An early and comprehensive paper on this topic

[28] Bruce C, Friedman H, Kraus M, Stanton G: The primate frontal eye field. In The Visual Neurosciences, Edited by Chalupa L, Werner J, MIT Press; 2004, 1429-1448. 2004.

- [29] Isa T, Yoshida M: Saccade control after V1 lesion revisited. Current Opinion in Neurobiology 2009, 19:608-614.

Showing that $\mathrm{V} 1$ lesion in monkeys makes animal unable to perform visually guided saccades for weeks

[30] Nakayama K, Mackeben M: Sustained and transient components of focal visual attention. Vision Research 1989, 29:631-47.

[31] Müller HJ, Rabbitt PM: Reflexive and voluntary orienting of visual attention: time course of activation and resistance to interruption. Journal of Experimental Psychology: Human Perception and Performance 1989, 15:315-330.

[32] Krummenacher J, Müller H, Heller D: Visual search for dimensionally redundant popout targets: Evidence for parallel-coactive processing of dimensions. Perception $\mathcal{E}$ Psychophysics 2001, 63:901-917.

- [33] Koene A, Zhaoping L: Feature-specific interactions in salience from combined feature contrasts: Evidence for a bottom-up saliency map in V1. Journal of Vision 2007, 7:article 6.

Showing that RTs in visual search for double-feature singletons are shorter than, or equal to, that predicted from a race model (due to statistical facilitation) between RTs for the corresponding single-feature singletons, and explaining how feature tuning in V1 neurons account for these findings by the V1 Saliency Hypothesis 
[34] Livingstone M, Hubel D: Anatomy and physiology of a color system in the primate visual cortex. The Journal of Neuroscience 1984, 4:309-56.

[35] Zhaoping L, Zhe L: Properties of V1 neurons tuned to conjunctions of visual features: application of the V1 saliency hypothesis to visual search behavior. PLoS One 2012, 7:e36223.

•• [36] Ben-Tov M, Donchin O, Ben-Shahar O, Segev R: Pop-out in visual search of moving targets in the archer fish. Nature Communications 2015, 6:article number 6476.

Visual search behavior and properties of OT neurons in archer fish

[37] Ben-Tov M, Kopilevich I, Donchin O, Ben-Shahar O, Giladi C, Segev R: Visual receptive field properties of cells in the optic tectum of the archer fish. Journal of neurophysiology 2013, 110:748-759.

[38] Tsvilling V, Donchin O, Shamir M, Segev R: Archer fish fast hunting maneuver may be guided by directionally selective retinal ganglion cells. European Journal of Neuroscience 2012, 35:436-444.

- [39] Hunter PR, Lowe AS, Thompson ID, Meyer MP: Emergent properties of the optic tectum revealed by population analysis of direction and orientation selectivity. The Journal of Neuroscience 2013, 33:13940-13945.

Showing that in zebra fish, some SC neurons construct new feature tuning that is absent from their retinal inputs

[40] Dhande OS, Huberman AD: Retinal ganglion cell maps in the brain: implications for visual processing. Current opinion in neurobiology 2014, 24:133-142.

[41] Vaney D, Peichl L, Wässle H, Illing RB: Almost all ganglion cells in the rabbit retina project to the superior colliculus. Brain research 1981, 212:447-453.

[42] Linden R, Perry V: Massive retinotectal projection in rats. Brain research 1983, 272:145149 .

[43] Hofbauer A, Dräger UC: Depth segregation of retinal ganglion cells projecting to mouse superior colliculus. Journal of Comparative Neurology 1985, 234:465-474.

-• [44] Berson D: Retinal ganglion cell types and their central projections. In The senses: a comprehensive reference, Edited by Masland R, Albright TD, Academic Press; 2008, volume 1, Vision I, 491-520. 2008.

A comprehensive review across animal species

•• [45] May PJ: The mammalian superior colliculus: laminar structure and connections. Progress in brain research 2006, 151:321-378.

A review with an emphasis on anatomy, including comparison between different animals

[46] Frost B, Nakayama K: Single visual neurons code opposing motion independent of direction. Science 1983, 220:744-745. 
[47] Wang L, Sarnaik R, Rangarajan K, Liu X, Cang J: Visual receptive field properties of neurons in the superficial superior colliculus of the mouse. The Journal of Neuroscience 2010, 30:16573-16584.

[48] Feinberg EH, Meister M: Orientation columns in the mouse superior colliculus. Nature 2015, 519:229-232.

[49] Dhande OS, Stafford BK, Lim JHA, Huberman AD: Contributions of retinal ganglion cells to subcortical visual processing and behaviors. Annual Review of Vision Science 2015, 1:291-328.

[50] Barlow H: Possible principles underlying the transformations of sensory messages. In Sensory Communication, Edited by Rosenblith WA, MIT Press; 1961, 217-234. 1961.

[51] Li Z: Different retinal ganglion cells have different functional goals. International Journal of Neural Systems 1992, 3:237-248.

[52] Masland RH: The neuronal organization of the retina. Neuron 2012, 76:266-280.

[53] Vaney DI, Sivyer B, Taylor WR: Direction selectivity in the retina: Symmetry and asymmetry in structure and function. Nature Reviews Neuroscience 2012, 13:194-208.

[54] Roska B, Meister M: The retina dissects the visual scene into distinct features. In The New Visual Neurosciences, Edited by Werner J, Chalupa L, MIT press, Cambridge, MA, USA; 2014, 163-182. 2014.

-• [55] Dacey D: Origins of perception: Retinal ganglion cell diversity and the creation of parallel visual pathways. In The Cognitive Neurosciences, 3rd edition, Edited by Gazzaniga MS, MIT press; 2004, 281-302. 2004.

Relating properties of the retinal ganglion cells and their central projections in monkeys

- [56] Baden T, Berens P, Franke K, Rosón MR, Bethge M, Euler T: The functional diversity of retinal ganglion cells in the mouse. Nature 2016, 529:345-350.

A detailed categorization of retinal ganglion cells in mouse

[57] Wylie DR, Gutierrez-Ibanez C, Pakan JM, Iwaniuk AN: The optic tectum of birds: mapping our way to understanding visual processing. Canadian Journal of Experimental Psychology/Revue canadienne de psychologie expérimentale 2009, 63:328-338.

[58] Bravo H, Pettigrew JD: The distribution of neurons projecting from the retina and visual cortex to the thalamus and tectum opticum of the barn owl, tyto alba, and the burrowing owl, speotyto cunicularia. Journal of Comparative Neurology 1981, 199:419-441.

[59] Chen Y, Naito J: Morphological properties of chick retinal ganglion cells in relation to their central projections. Journal of Comparative Neurology 2009, 514:117-130.

- [60] Li DP, Xiao Q, Wang SR: Feedforward construction of the receptive field and orientation selectivity of visual neurons in the pigeon. Cerebral Cortex 2007, 17:885-893.

Showing that pigeon OT cells construct additional feature tunings from retinal inputs 
[61] Hubel DH, Wiesel TN: Receptive fields, binocular interaction and functional architecture in the cat's visual cortex. The Journal of physiology 1962, 160:106-154.

[62] Mangini NJ, Pearlman AL: Laminar distribution of receptive field properties in the primary visual cortex of the mouse. The Journal of comparative neurology 1980, 193:203-222.

[63] Niell CM, Stryker MP: Highly selective receptive fields in mouse visual cortex. The Journal of Neuroscience 2008, 28:7520-7536.

[64] Sun W, Tan Z, Mensh BD, Ji N: Thalamus provides layer 4 of primary visual cortex with orientation- and direction-tuned inputs. Nature neuroscience 2016, 19:308-315.

[65] Wilson P: The organization of the visual hyperstriatum in the domestic chick. ii. receptive field properties of single units. Brain research 1980, 188:333-345.

[66] Pettigrew JD, Konishi M: Neurons selective for orientation and binocular disparity in the visual wulst of the barn owl (tyto alba). Science 1976, 193:675-678.

[67] Hodos W, Karten H: Visual intensity and pattern discrimination deficits after lesions of the optic lobe in pigeons; pp. 180-194. Brain, behavior and evolution 1974, 9:180-194.

[68] Prusky G, Douglas R: Characterization of mouse cortical spatial vision. Vision research 2004, 44:3411-3418.

[69] Glickfeld LL, Histed MH, Maunsell JH: Mouse primary visual cortex is used to detect both orientation and contrast changes. The Journal of Neuroscience 2013, 33:19416-19422.

[70] Sridharan D, Schwarz JS, Knudsen EI: Selective attention in birds. Current Biology 2014, 24:R510-R513. 